\title{
Influence of Donor, Recipient, and Male Partner Body Mass index on Pregnancy Rates in Oocyte Donation Cycles
}

\author{
Carolina Rubio ${ }^{1}$, Rita Vassena ${ }^{2}$, Désirée García ${ }^{3}$, Valérie Vernaeve ${ }^{2,3}$, José Ignacio Madero ${ }^{1}$ \\ ${ }^{1}$ Clínica EUGIN, Bogotá, Colombia \\ ${ }^{2}$ Clínica EUGIN, Barcelona 08029, Spain \\ ${ }^{3}$ Fundació Privada EUGIN, Barcelona 08029, Spain \\ Presented at the Fertility 2014 Conference, Leeds - UK
}

\begin{abstract}
Objective: A high body mass index (BMI) has been shown to associate with negative reproductive outcomes. Women with high BMI have in general lower chances of getting pregnant as well as higher risk of pregnancy complications. Several studies have described in the past the relationship between high BMI and the pregnancy outcome, however, some of them have a small sample size or fail to control for variables associated with a diminished probability of pregnancy. In the present study, we aim to analyze the role of the BMI of all parties involved in oocyte donation cycles (that is: the oocyte donor, the recipient woman, and the male partner) on pregnancy outcomes.
\end{abstract}

Methods: This study includes 1092 oocyte donation cycles. Inclusion criteria were: fertilization by ICSI, frozen semen, transfer of 2 embryos at day 3 of in vitro development. For statistical analysis, BMI was divided in: low weight $(<20 \mathrm{~kg} / \mathrm{m} 2)$, normal $(20-24 \mathrm{~kg} / \mathrm{m} 2)$, overweight $(25-29 \mathrm{~kg} / \mathrm{m} 2)$ and obesity $(\geq 30 \mathrm{~kg} / \mathrm{m} 2)$. Quantitative and categorical variables were assessed by squared-Chi test and one-way ANOVA. The association between the BMI (recipient, oocyte donor and partner) and pregnancy rate was assessed by multivariate logistic regression.

Results: Laboratory outcomes and pregnancy rates do not differ among the different BMI categories of recipient, oocyte donor or partner. After adjusted analyses (for oocyte donor age, for laboratory outcomes and for age and BMI of all the parties for pregnancy outcomes), no difference was found either.

Conclusion: In oocyte donation cycles, where donors BMI is by law mandated to be in the $18-30$ range, the pregnancy rate of the oocyte recipient does not seem to be affected by the BMI of any of the parties involved.

Keywords: Body mass index, In vitro fertilization, Intracytoplasmic sperm injection, Embryo quality, Male obesity, Female obesity.

\section{INTRODUCTION}

A high body mass index (BMI) in women has been shown to negatively affect their reproductive outcomes, as these women have both lower chances of getting pregnant and higher chances of pregnancy complications (Garcia et al., 2014). On one hand, a BMI higher than $25 \mathrm{~kg} / \mathrm{m} 2$ has been reported to diminish both the number and quality of the MII oocytes obtained after controlled ovarian stimulation (Esinler et al., 2008, Wittemer et al., 2000), which in turn results in embryos of lower quality (Zhang et al., 2010). Even when the female gamete is of high quality, such as in oocyte donation cycles, high BMI in the recipient is also associated with an increased abortion rate (Bellver et al., 2003) and a lower ongoing pregnancy rates (Bellver et al., 2007).

The endocrine changes associated with obesity can impact endometrial receptivity; obese women have more an- drogen receptors in their endometrium (Okon et al., 1998), and have altered levels of androstenedione, which depresses endometrial receptivity (Tuckerman et al., 2000) and, through a decreased expression of glycodelin, increases their susceptibility to abortion (Salim et al., 2007).

Moreover, hyperinsulinemia associated with obesity decreases IGFBP1 levels in the uterus, an important molecule for embryonic adhesion (Butzow et al., 2000, Fowke et al., 2010). Finally, an increased concentration of leptin in the uterine environment in obese women has also been related to alteration in trophoblast invasion, implantation, and an increased risk of abortion (Castellucci et al., 2000).

A lack of association between recipient BMI and pregnancy rate in oocyte donation cycles has been reported (Styne-Gross et al., 2005); these results were confirmed in a meta-analysis (Jungheim et al., 2013); however, the authors cautions against the over interpretation of their results, as they proceed from small and heterogeneous studies. A large study which analyzes reproductive outcomes in oocyte donation cycles, including BMIs of both donors and recipient, and also taking into account the increasingly important BMI of the male partner, is still lacking.

The relationship between male BMI and reproductive outcomes is much less studied; obese man have worse reproductive results with natural conceptions; male obesity is associated with low levels of androgens and high levels of estrogens, which result in hypergonadotropic hypogonadism associated with hypoandrogenemia (Colaci et al., 2012). Hyperestrogenism causes an alteration in the pulsatile release of GnRH (Hammoud et al., 2008). Both high weight and obesity in males have been associated to poor semen quality (Colaci et al., 2012, Hammoud et al., 2008) as well as to a higher proportion of DNA fragmentation in semen (Hammoud et al., 2008; Colaci et al., 2012), and infertility (Sallmen et al., 2006).

However, the relationship between BMI in male and reproductive outcomes in assisted reproduction has not been studied in depth and there is no general consensus (Thomsen et al., 2014)

The objective of the present study was to investigate the effect of the BMI of all parties involved on the pregnancy rates of women recipients of oocyte donation.

\section{MATERIAL AND METHODS}

This is a retrospective analysis of anonymized data from 1092 oocyte donation cycles between November 2010 and August 2013 at a large reproductive centre. This study did not require the approval of an Ethics Committee because the data were anonymous. Nevertheless, permission to conduct the study was sought and obtained from the Institutional Review Board of Clínica EUGIN.

\section{Recipient characteristics}

The indications for oocyte donation were failed IVF cycles with own oocytes, low ovarian reserve, poor oocyte 
quality, genetic or chromosomal abnormalities transmissible to offspring and spontaneous or iatrogenic menopause. Women with menstrual cycles received a depot dose of $\mathrm{GnRH}$ agonist to suppress the pituitary. The estrogen endometrial preparation was started in the following menstrual cycle. An increasing dose of estrogens was administered orally or transdermally (from 2 to $6 \mathrm{mg}$, or from 75 to $150 \mathrm{mg}$, respectively) for a period of 15-40 days (average: 20.4; SD: 8 ; median: 17). This length of time was kept in all cases below 49 days, the limit above which a significant decrease in uterine receptivity has been demonstrated (Soares et al. , 2005).

\section{Donor characteristics}

All oocyte donors (age 18-35 years) had normally appearing ovaries at transvaginal ultrasound, adequate ovarian reserve as evidenced by an antral follicular count above 10 , and displayed a correct response to ovarian stimulation, i.e. a progressive and gradual increase in follicular sizes, concordant with FSH administration. All donors were stimulated with exogenous gonadotrophins, while the pituitary suppression was based on two possible protocols: $\mathrm{GnRH}$ antagonists fixed from the sixth day of ovarian stimulation (most of the cycles) or GnRH agonists starting in the second phase of the preceding menstrual cycle. Regardless of the stimulation protocol, ovulation was triggered when three or more follicles $\geq 18 \mathrm{~mm}$ diameter were present on the ovaries. Ovulation trigger was performed with either $0.2 \mathrm{mg}$ of the GnRH agonist Triptorelin (Decapeptyl Ipsen, Pharma Biotech, France) or 250 mg hCG (Ovitrelle, Merck, Germany) depending on the stimulation protocol. Oocyte collection was performed 36 hours after triggering by means of ultrasound-guided transvaginal follicular aspiration.

\section{Partner characteristics}

The inclusion criterion for this study was oocyte donation cycles with ICSI as a fertilization method. Exclusion criteria were semen coming from a sperm donor, semen obtained by testicular biopsy and semen frozen prior to treatment for a medical condition. All patients were requested to maintain 3-5 days of abstinence before providing the semen sample used for evaluation and fertilization. All sperm samples were analyzed (volume, concentration, motility) using the standard laboratory procedures of the World Health Organization (WHO, 2010).

\section{Statistical analysis}

For the statistical analysis, the following BMI categories were created $(\mathrm{kg} / \mathrm{m} 2)$ : underweight $(<20)$, normal weight (20-24), overweight (25-29) and obesity ( $\geq 30)$. Quantitative and categorical variables were assessed by squared-Chi test or one-way ANOVA, respectively. The association between the BMI categories (recipient, oocyte donor and partner) and the biochemical pregnancy rate was assessed by multivariate logistic regression (adjusting for recipient, donor, and partner age as well as recipient and donor BMI). The association between the donor BMI categories and the laboratory outcomes (number of COC, MII, and 2PN) was assessed by linear regression (adjusting for donor age). All analyses were performed using SPSS 20.

\section{RESULTS}

A total of 1092 cycles were included. Figure 1 shows the BMI distribution by recipient, oocyte donor, and partner.

Table 1: Laboratory outcomes. Univariate analysis ( $P$ One-way ANOVA)
\begin{tabular}{|l|c|c|c|c|c|}
\hline \multicolumn{1}{|c|}{ Donor BMI } & $<\mathbf{2 0}$ & $\mathbf{2 0 - 2 5}$ & $\mathbf{2 5 - 3 0}$ & $\mathbf{2 3 0}$ & $\boldsymbol{P}$ \\
\hline $\begin{array}{l}\text { Number of COC } \\
{[\text { median (IQR;Max-Min) }]}\end{array}$ & $8(2 ; 5-16)$ & $8(4 ; 4-26)$ & $8(3 ; 4-22)$ & 3 & 0.50 \\
\hline $\begin{array}{l}\text { Number of MII } \\
{[\text { median (IQR;Max-Min) }]}\end{array}$ & $6(1 ; 4-12)$ & $6(1 ; 4-12)$ & $6(1 ; 4-11)$ & $6(1 ; 6-7)$ & 0.88 \\
\hline $\begin{array}{l}\text { Number of 2PN } \\
{[\text { median (IQR;Max-Min) }]}\end{array}$ & $5(2 ; 2-10)$ & $5(2 ; 2-10)$ & $5(2 ; 2-8)$ & $6(4 ; 2-7)$ & 0.74 \\
\hline
\end{tabular}

\begin{tabular}{|c|c|c|c|}
\hline & & Effect & $\boldsymbol{P}$ \\
\hline \multirow{3}{*}{ Number of COC } & Donor $\mathrm{BMI}<20$ & -0.126 & 0.53 \\
\hline & Donor $\mathrm{BMI} \geq 25$ & 0.275 & 0.21 \\
\hline & Donor age & -0.012 & 0.49 \\
\hline \multirow{3}{*}{ Number of MII } & Donor BMI<20 & 0.083 & 0.39 \\
\hline & Donor $\mathrm{BMI} \geq 25$ & -0.010 & 0.92 \\
\hline & Donor age & 0.012 & 0.18 \\
\hline \multirow{3}{*}{ Number of $2 \mathrm{PN}$} & Donor $\mathrm{BMI}<20$ & -0.020 & 0.86 \\
\hline & Donor $\mathrm{BMI} \geq 25$ & 0.115 & 0.34 \\
\hline & Donor age & -0.003 & 0.80 \\
\hline
\end{tabular}


Table 3. Pregnancy outcomes. Univariate analysis ( $P \mathrm{Chi}^{2}$ test)

\begin{tabular}{|l|c|c|c|c|}
\hline \multicolumn{1}{|c|}{ Recipient BMI } & $<\mathbf{2 0}$ & $\mathbf{2 0 - 2 4}$ & $\mathbf{2 5 - 2 9}$ & $\mathbf{P}$ \\
\hline \multirow{3}{*}{ BhCG- } & 85 & 318 & 116 & 54 \\
\hline \multirow{3}{*}{ BhCG+ } & $48.3 \%$ & $52.9 \%$ & $54.0 \%$ & $54.0 \%$ \\
\hline \multirow{2yyyy}{*}{ Oocyte donor BMI } & 91 & 283 & 99 & 46 \\
\hline \multirow{2}{*}{ BhCG- } & $51.7 \%$ & $47.1 \%$ & $46.0 \%$ & $46.0 \%$ \\
\hline \multirow{2}{*}{ BhCG+ } & $<\mathbf{2 0}$ & $\mathbf{2 0 - 2 4}$ & $\mathbf{2 5 - 2 9}$ & $\geq \mathbf{3 0}$ \\
\hline \multirow{2}{*}{ Partner BMI } & 129 & 339 & 103 & 2 \\
\hline \multirow{2}{*}{ BhCG- } & $51.4 \%$ & $52.8 \%$ & $53.6 \%$ & $28.6 \%$ \\
\hline \multirow{2}{*}{ BhCG+ } & 122 & 303 & 89 & 5 \\
\cline { 2 - 5 } & $48.6 \%$ & $47.2 \%$ & $46.4 \%$ & $71.4 \%$ \\
\hline
\end{tabular}

Table 4. Pregnancy outcomes. Multivariate analysis (Reference BMI category: 20-24).

\begin{tabular}{|l|c|c|c|c|}
\hline & \multirow{2}{*}{$\mathbf{O R}$} & \multicolumn{2}{|c|}{ 95\% CI OR } & \multirow{2}{*}{$\boldsymbol{P}$} \\
\cline { 3 - 5 } & & Lower & Upper \\
\hline Recipient BMI $<20$ & 1.18 & 0.84 & 1.66 & 0.80 \\
\hline Recipient BMI 25-39 & 0.98 & 0.72 & 1.35 & 0.34 \\
\hline Recipient BMI $\geq 30$ & 1.01 & 0.66 & 1.56 & 0.92 \\
\hline Recipient age & 0.98 & 0.96 & 1.01 & 0.96 \\
\hline Donor BMI $<20$ & 1.03 & 0.77 & 1.38 & 0.86 \\
\hline Donor BMI 25-39 & 0.98 & 0.71 & 1.36 & 0.92 \\
\hline Donor BMI $\geq 30$ & 2.64 & 0.50 & 14.02 & 0.25 \\
\hline Donor age & 1.00 & 0.97 & 4.98 & 0.74 \\
\hline Partner BMI <20 & 2.20 & 0.97 & 1.17 & 0.06 \\
\hline Partner BMI 25-39 & 0.90 & 0.70 & 1.48 & 0.45 \\
\hline Partner BMI $\geq 30$ & 0.97 & 0.63 & 1.02 & 0.87 \\
\hline Partner age & 1.00 & 0.98 & & 0.06 \\
\hline
\end{tabular}

\section{Laboratory outcomes}

Laboratory outcomes, i.e. number of cumulus-oocyte complexes, mature (MII) and fertilized (2PN) oocytes recovered from the follicular punction, do not differ among the different categories of oocyte donor BMI, as shown by univariate analysis (Table 1 ). After adjusting for donor age, and taking normal BMI (20-24) as reference, the association between donor BMI and laboratory outcomes remains no statistically significant (Table 2).

\section{Pregnancy outcomes}

Pregnancy rates (defined as a positive BHCG blood test taken 14 days after embryo transfer), do not differ among categories of recipient, oocyte donor, or partner BMI at univariate analysis (Table 3 ). In the same way, the multivariate analysis (Table 4) shows that, after adjusting for recipient and donor age, and taking normal BMI (20-24) as reference, there is no effect of recipient, donor or partner BMI on pregnancy outcomes.

\section{DISCUSSION}

\section{BMI and pregnancy}

The impact of BMI on fertility has been studied extensively, focusing mostly o the effect of high BMI (obesity) on fertilization rate, implantation, and pregnancy loss (Jungheim et al., 2013, Luke et al., 2011, Schliep et al., 2014, Sobaleva \& El-Toukhy, 2011, Styne-Gross et al., 2005). While some authors describe lower oocyte and embryo quality in obese patients (Bu et al., 2013, Valckx et al., 2012), others did not find this association (Wang et al., 2000). A report indicates the presence of fewer mature oocytes after controlled ovarian stimula- 
Figure 1. BMI distribution for each of the parties involved in an oocyte donation cycle.

\section{A). Recipient}

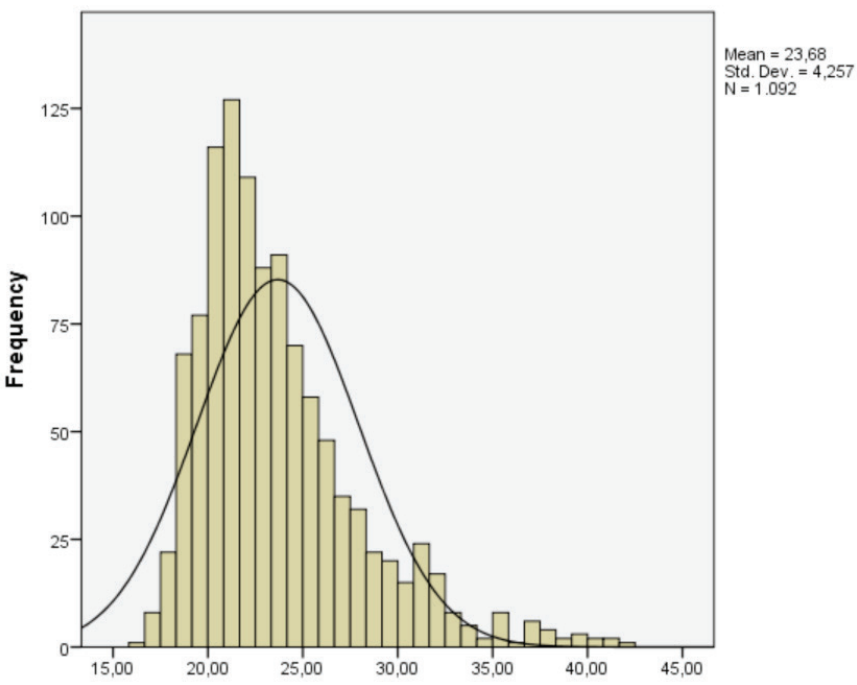

B). Oocyte donor

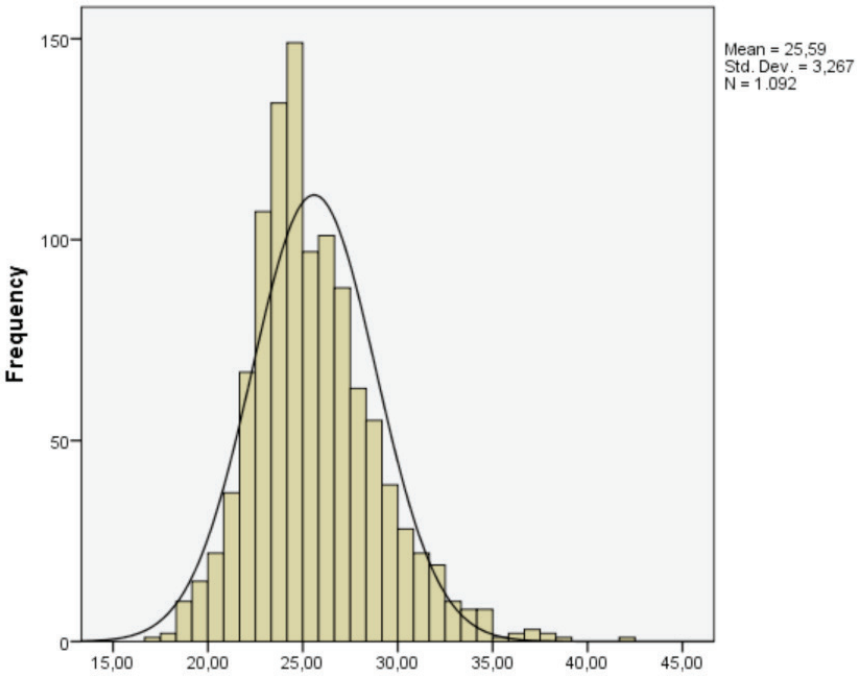

C). Partner

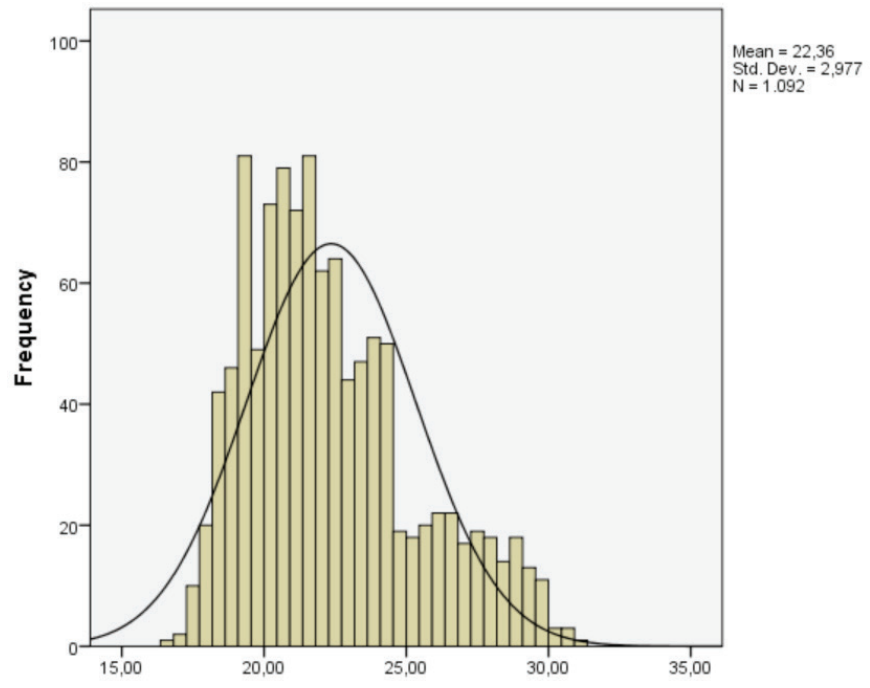

tion in obese patients, lower fertilization rate, and lower number of transferred embryos (Bellver et al., 2010). A study evaluating 6500 in vitro fertilization cycles with patient own oocytes found that obese women had significantly higher gonadotropin consumption during controlled ovarian stimulation (Bellver et al., 2010). Moreover, the researchers found lower implantation, pregnancy, and live birth rates as the BMI increased. However, oocyte quality, evaluated by embryo quality on day 2 and 3 of development, was not affected by the patient BMI in this study. Taken together, these results suggest that obesity can either alter embryo quality by mechanisms which are not reflected in embryo morphology, or that the effect is mostly exerted on the endometrium.

The donor-recipient model allows for the uncoupling of the two factors that have been mostly suspected of being altered by high BMI: oocyte quality and endometrial receptivity.

\section{BMI in donation cycles}

We did not find any relationship between BMI of recipient and the partner and pregnancy rate, and this is in agreement with what found by other researchers when analyzing the effect of body mass index (BMI) on implantation, pregnancy, and incidence of spontaneous miscarriage using the donor oocyte recipient model (Styne-Gross et al., 2005, Wittemer et al., 2000). Similar results were found in the study by Schliep et al. (2014), where they reported no significant associations between male, female, or couple BMI and fertilization rate, embryo score, clinical pregnancy, or live birth after adjustment for relevant confounders. A study evaluating 536 oocyte recipients (Styne-Gross et al., 2005) did not found significant differences in implantation rate, ongoing pregnancy or abortion rate across recipient BMI categories. These results confirmed what found in a previous, smaller study (Bellver et al., 2003). A subsequent larger study by the same group (Bellver et al., 2007) comprising 2656 recipient, 122 of which obese, found a tendency to a lower implantation rate and pregnancy rate, as well as higher rates of ectopic pregnancy and abortion as the recipient BMI increased. Moreover, there was a significantly lower ongoing pregnancy rate for started cycle in obese women compared to those of normal BMI. Finally, Dessolle et al. (2009) evaluated 450 donation cycles, and found a significant drop in pregnancy rate in recipient with BMI over 30 $\mathrm{kg} / \mathrm{m} 2$, regardless of age.

In our study, we found that when the embryo is derived from a donor of $\mathrm{BMI}<30$, i.e. not obese, there is no effect of the recipient BMI on pregnancy rates, which indicates that the worse reproductive outcomes in obese patients using their own oocytes should be due to oocyte quality rather than an effect on the endometrium.

\section{BMI in the male partner}

The relationship between semen characteristics and male BMI is unclear; morphological sperm defects have been related to high BMI (Hammoud et al., 2008) while more recent studies did not find a relationship between male BMI and seminal parameters and sperm binding ability, in a population of male with low or moderate idiopathic infertility (Sermondade et al., 2013, Thomsen et al., 2014), as well as pregnancy rates (Isa et al. , 2014, Thomsen et al., 2014). Interestingly, pregnancy rates of obese patients increased after ICSI but not after conventional IVF (Merhi et al. , 2013). However, a study by Colaci et al. (2012) reports that male obesity might have a negative effect on pregnancy rates after ICSI as well. The cycles analyzed in this study were all carried out with ICSI as fertilization technique, and in agreement with the study by 
Merhi we did not find an effect of male BMI on pregnancy rates, strengthening the hypothesis that the worse reported outcome in conventional IVF might be due to functional alteration in the sperm such as motility or the ability to penetrate the zona pellucida.

\section{CONCLUSION}

In conclusion, we found that a high BMI in oocyte recipient and her male partner do not impact pregnancy rates when the oocytes come from young women with normal BMI, and when the fertilization technique is ICSI. We suggest that both these factors might ease some of the effect of high BMI, such as low oocyte quality and altered sperm function, thus allowing for high pregnancy rates across the BMI spectrum. As pregnancy complications are more frequent in obese patients, counseling should be extended to high BMI women regardless of their chance to attain a pregnancy.

\section{ACKNOWLEDGEMENTS}

The authors wish to thank Francesc Figueras for statistical support.

\section{CONFLICT OF INTERESTS}

No conflict of interest have been declared.

\section{Corresponding author:}

Rita Vassena

Clínica EUGIN,

Barcelona, Spain.

E-mail: rvassena@eugin.es

\section{REFERENCES}

Bellver J, Ayllon Y, Ferrando M, Melo M, Goyri E, Pellicer A, Remohi J, Meseguer M. Female obesity impairs in vitro fertilization outcome without affecting embryo quality. Fertil Steril. 2010;93:447-54.

Bellver J, Melo MA, Bosch E, Serra V, Remohi J, Pellicer A. Obesity and poor reproductive outcome: the potential role of the endometrium. Fertil Steril. 2007;88:446-51.

Bellver J, Rossal LP, Bosch E, Zuniga A, Corona JT, Melendez F, Gomez E, Simon C, Remohi J, Pellicer A. Obesity and the risk of spontaneous abortion after oocyte donation. Fertil Steril. 2003;79:1136-40.

Bu Z, Dai W, Guo Y, Su Y, Zhai J, Sun Y. Overweight and obesity adversely affect outcomes of assisted reproductive technologies in polycystic ovary syndrome patients. International journal of clinical and experimental medicine. Int J Clin Exp Med. 2013;6:991-5.

Butzow TL, Lehtovirta M, Siegberg R, Hovatta O, Koistinen $R$, Seppala M, Apter D. The decrease in luteinizing hormone secretion in response to weight reduction is inversely related to the severity of insulin resistance in overweight women. J Clin Endocrinol Metab. 2000;85:3271-5.

Castellucci M, De Matteis R, Meisser A, Cancello R, Monsurro V, Islami D, Sarzani R, Marzioni D, Cinti S, Bischof P. Leptin modulates extracellular matrix molecules and metalloproteinases: possible implications for trophoblast invasion. Mol Hum Reprod. 2000;6:951-8.

Colaci DS, Afeiche M, Gaskins AJ, Wright DL, Toth TL, Tanrikut C, Hauser R, Chavarro JE. Men's body mass index in relation to embryo quality and clinical outcomes in couples undergoing in vitro fertilization. Fertil Steril. 2012;98:1193-9 e1.
Dessolle L, Darai E, Cornet D, Rouzier R, Coutant C, Mandelbaum J, Antoine JM. Determinants of pregnancy rate in the donor oocyte model: a multivariate analysis of 450 frozen-thawed embryo transfers. Hum Reprod. 2009;24:3082-9.

Esinler I, Bozdag G, Yarali H. Impact of isolated obesity on ICSI outcome. Reprod Biomed Online. 2008;17:583-7.

Fowke JH, Matthews CE, Yu H, Cai Q, Cohen S, Buchowski MS, Zheng W, Blot WJ. Racial differences in the association between body mass index and serum IGF1, IGF2, and IGFBP3. Endocr Relat Cancer. 2010;17:51-60.

Garcia D, Vassena R, Trullenque M, Rodriguez A, Vernaeve V. Fertility knowledge and awareness in oocyte donors in Spain. Patient Educ Couns. 2015;98:96-101.

Hammoud AO, Wilde N, Gibson M, Parks A, Carrell DT, Meikle AW. Male obesity and alteration in sperm parameters. Fertil Steril. 2008;90:2222-5.

Isa AM, Abu-Rafea B, Alasiri SA, Binsaleh $\mathrm{S}$, Ismail KH, Vilos GA. Age, body mass index, and number of previous trials: are they prognosticators of intra-uterine-insemination for infertility treatment? Int J Fertil Steril. 2014;8:255-60.

Jungheim ES, Schon SB, Schulte MB, DeUgarte DA, Fowler SA, Tuuli MG. IVF outcomes in obese donor oocyte recipients: a systematic review and meta-analysis. Hum Reprod. $2013 ; 28: 2720-7$

Luke B, Brown MB, Stern JE, Missmer SA, Fujimoto VY, Leach R, Group SW. Female obesity adversely affects assisted reproductive technology (ART) pregnancy and live birth rates. Hum Reprod. 2011;26:245-52.

Merhi ZO, Keltz J, Zapantis A, Younger J, Berger D, Lieman $\mathrm{HJ}$, Jindal SK, Polotsky AJ. Male adiposity impairs clinical pregnancy rate by in vitro fertilization without affecting day 3 embryo quality. Obesity. 2013;21:1608-12.

Okon MA, Laird SM, Tuckerman EM, Li TC. Serum androgen levels in women who have recurrent miscarriages and their correlation with markers of endometrial function. Fertil Steril. 1998;69:682-90.

Salim R, Miel J, Savvas M, Lee C, Jurkovic D. A comparative study of glycodelin concentrations in uterine flushings in women with subseptate uteri, history of unexplained recurrent miscarriage and healthy controls. Eur J Obstet Gynecol Reprod Biol. 2007;133:76-80

Sallmen M, Sandler DP, Hoppin JA, Blair A, Baird DD. Reduced fertility among overweight and obese men. Epidemiology. 2006;17:520-3.

Schliep KC, Mumford SL, Ahrens KA, Hotaling JM, Carrell DT, Link M, Hinkle SN, Kissell K, Porucznik CA, Hammoud AO. Effect of male and female body mass index on pregnancy and live birth success after in vitro fertilization. Fertil Steril. 2015; 103:388-95.

Sermondade N, Dupont C, Faure C, Boubaya M, Cedrin-Durnerin I, Chavatte-Palmer $P$, Sifer C, Levy R. Body mass index is not associated with sperm-zona pellucida binding ability in subfertile males. Asian J Androl. 2013;15:626-9.

Soares SR, Troncoso C, Bosch E, Serra V, Simon C, Remohi 
J, Pellicer A. Age and uterine receptiveness: predicting the outcome of oocyte donation cycles. J Clin Endocrinol Metab. 2005; 90:4399-404.

Sobaleva S, El-Toukhy T. The impact of raised BMI on the outcome of assisted reproduction: current concepts. J Obstet Gynaecol. 2011;31:561-5.

Styne-Gross A, Elkind-Hirsch K, Scott RT, Jr. Obesity does not impact implantation rates or pregnancy outcome in women attempting conception through oocyte donation. Fertil Steril. 2005;83:1629-34.

Thomsen L, Humaidan P, Bungum L, Bungum M. The impact of male overweight on semen quality and outcome of assisted reproduction. Asian J Androl. 2014;16:749-54.

Tuckerman EM, Okon MA, Li T, Laird SM. Do androgens have a direct effect on endometrial function? An in vitro study. Fertil Steril. 2000;74:771-9.

Valckx SD, De Pauw I, De Neubourg D, Inion I, Berth M, Fransen E, Bols PE, Leroy JL. BMI-related metabolic com- position of the follicular fluid of women undergoing assisted reproductive treatment and the consequences for oocyte and embryo quality. Hum Reprod. 2012;27:3531-9.

Wang JX, Davies M, Norman RJ. Body mass and probability of pregnancy during assisted reproduction treatment: retrospective study. BMJ. 2000;321:1320-1.

WHO. WHO Laboratory Manual for the Examination and Processing of Human Semen, 5th edn. Geneva:World Health Organization. 2010.

Wittemer C, Ohl J, Bailly M, Bettahar-Lebugle K, Nisand I. Does body mass index of infertile women have an impact on IVF procedure and outcome? J Assist Reprod Genet. $2000 ; 17: 547-52$

Zhang $D$, Zhu $Y$, Gao $H$, Zhou B, Zhang $R$, Wang $T$, Ding G, Qu F, Huang H, Lu X. Overweight and obesity negatively affect the outcomes of ovarian stimulation and in vitro fertilisation: a cohort study of 2628 Chinese women. Gynecol Endocrinol. 2010;26: 325-32. 